\title{
Nanobubbles for enhanced ultrasound imaging of tumors
}

This article was published in the following Dove Press journal:

International Journal of Nanomedicine

21 February 2012

Number of times this article has been viewed

\author{
Tinghui Yin $1 *$ \\ Ping Wang ${ }^{*}$ \\ Rongqin Zheng' \\ Bowen Zheng' \\ Du Cheng ${ }^{2}$ \\ Xinling Zhang' \\ Xintao Shuai ${ }^{2}$ \\ 'Department of Medical Ultrasonic, \\ Third Affiliated Hospital, ${ }^{2}$ PCFM \\ Laboratory of the Ministry of \\ Education, School of Chemistry and \\ Chemical Engineering, Sun Yat-Sen \\ University, Guangzhou, People's \\ Republic of China \\ *These authors contributed equally \\ to this work
}

\begin{abstract}
The fabrication and initial applications of nanobubbles (NBs) have shown promising results in recent years. A small particle size is a basic requirement for ultrasound contrastenhanced agents that penetrate tumor blood vessel pores to allow for targeted imaging and therapy. However, the nanoscale size of the particles used has the disadvantage of weakening the imaging ability of clinical diagnostic ultrasound. In this work, we fabricated a lipid NBs contrast-enhanced ultrasound agent and evaluated its passive targeting ability in vivo. The results showed that the NBs were small $(436.8 \pm 5.7 \mathrm{~nm})$, and in vitro ultrasound imaging suggested that the ultrasonic imaging ability is comparable to that of microbubbles (MBs). In vivo experiments confirmed the ability of NBs to passively target tumor tissues. The NBs remained in the tumor area for a longer period because they exhibited enhanced permeability and retention. Direct evidence was obtained by direct observation of red fluorescence-dyed NBs in tumor tissue using confocal laser scanning microscopy. We have demonstrated the ability to fabricate NBs that can be used for the in vivo contrast-enhanced imaging of tumor tissue and that have potential for drug/gene delivery.
\end{abstract}

Keywords: phospholipids, ultrasound, contrast agent, tumor-targeted

\section{Introduction}

Tumors induce angiogenesis to increase their nutrient and oxygen supply, enabling their rapid growth. ${ }^{1}$ However, the blood vessels in tumors are leaky and defective, with large pore cutoff sizes, and the endothelial cells are misaligned or have large fenestrations. ${ }^{2}$ Moreover, tumors have poor lymphatic drainage compared with normal tissues. ${ }^{2,3}$ All of these features allow for the delivery of drugs and gene carriers, such as liposomes, polymer micelles/vesicles and other macromolecules, to the tumor tissue; this ability is known as the enhanced permeability and retention (EPR) effect., ${ }^{4,5}$

The EPR effect of tumor blood vessels enables the design of targeted treatment options such as the use of small, negatively charged and PEGylated agents. ${ }^{6}$ One of the basic requirements for therapeutic or imaging agents to be able to leak through tumor pores for tumor-targeted therapy and imaging is a small particle size.

The application of microbubbles (MBs) in contrast-enhanced ultrasound (CEUS) has become an indispensable part of clinical ultrasonography, ${ }^{7}$ and molecular imaging via ultrasound has recently attracted significant attention. ${ }^{8}$ MBs help to enhance the specificity and sensitivity of imaging for various types of diseases, especially with tumors. ${ }^{9}$ Other advantages of using MBs include a lower cost of contrast agents, an opportunity for real-time observation and the elimination of the exposure to radiation. ${ }^{10}$ 
In the development of molecular imaging, the fundamental enabling technology for ultrasonic molecular imaging is the targeted CEUS agent. ${ }^{11}$ However, compared with contrast agents for computed tomography $(\mathrm{CT})$ and magnetic resonance imaging (MRI), MBs are much larger $(2-8 \mu \mathrm{m})$. Therefore, when used in diagnostic ultrasound, MBs can become trapped in the blood pool after intravenous injection. Consequently, most research on targeted MBs has been limited to studies involving diseases of the cardiovascular system, such as inflammation, arteriosclerosis, and thrombus formation. ${ }^{12-15} \mathrm{MBs}$ pose severe limitations in tumor-targeted imaging due to their large diameters.

Recently developed nanoscale bubbles (nanobubbles [NBs]) are promising contrast agents for extravascular ultrasonic imaging. Nanoscale ultrasound contrast agents with various shells (polymers or phospholipids) and cores (gas, liquid, or solid) have been fabricated and exhibit good contrast enhancement. Based on several in vitro ${ }^{16-19}$ and in vivo ${ }^{20}$ studies, phospholipid-shell and gas-core NBs have shown optimal contrast enhancement abilities. However, research on NBs is still in the initial stages. ${ }^{21,22}$ Moreover, in vivo studies have focused on the contrast enhancement abilities of these agents in only normal organs or in tumors, and their passive tumor-targeted potential has not yet been explored. Whether NBs can pass through the endothelial gaps of tumors and maintain a high imaging quality is unknown.

In this study, phospholipids were used to form the membrane for the fabrication of NBs, which then demonstrated an ultrasonic imaging ability similar to that of MBs. Scanning electron microscopy (SEM) was used to evaluate the morphology of the NBs. In vivo experiments confirmed the passive targeting ability of NBs in tumor tissues. The NBs remained in the tumor area for a longer period than MBs, and a high imaging quality was detected using in vivo tumor ultrasound imaging. Red fluorescent dye-labeled NBs were observed to remain in tumor tissues, as evaluated using confocal laser scanning microscopy, and this finding further supports the conclusion that NBs are passively targeted to tumor tissue.

\section{Material and methods Materials}

The phospholipids used in the fabrication of the NBs included 1,2-dipalmitoyl-sn-glycero-3-phosphocholine (DPPC; Mw, 734.05), 1,2-distearoyl-sn-glycero-3-phosphoethanolamineN-[biotinyl(polyethylene glycol)2000] (PEG2000-DSPE; Mw, 3016.81) and 1,2-dipalmitoyl-sn-glycero-3-phosphate (DPPA; Mw, 670.88), which was in powder form (Avanti
Polar Lipids Inc, Alabaster, AL) and used without further purification. Pluronic F-68 and glycerol were purchased from Sigma-Aldrich (St Louis, $\mathrm{MO})$. Octafluoropropane $\left(\mathrm{C}_{3} \mathrm{~F}_{8}\right)$ gas was purchased from the R\&D Center for Specialty Gases at the Research Institute of Physical and Chemical Engineering of Nuclear Industry (Tianjin, China). The fluorescent probes, DiI and Hoechst 33342, were purchased from Beyotime (Haimen, China).

\section{Synthesis of the bubbles}

NBs were prepared using a thin-film hydration-sonication method. ${ }^{22}$ Briefly, all phospholipids (18 mg DPPC, $3.5 \mathrm{mg}$ PEG2000-DSPE, and $1 \mathrm{mg}$ DPPA) were dissolved in $4 \mathrm{~mL}$ of chloroform (a small amount of the fluorescent membrane probe DiI was added for confocal laser scanning microscopy) and transferred into a 9-cm culture dish to form a thin phospholipid film by natural evaporation in a fume hood. The material was then hydrated with $4 \mathrm{~mL}$ of hydration liquid, which consisted of $10 \%$ glycerol (v/v) and $2 \mathrm{mg} / \mathrm{mL}$ Pluronic F-68, at $37^{\circ} \mathrm{C}$ in a shaking incubator for 1 hour to prepare liposomes. The liposomal suspension was transferred to a $50-\mathrm{mL}$ centrifuge tube, and the air above the liquid was replaced with $\mathrm{C}_{3} \mathrm{~F}_{8}$ gas using a long, fine needle and a $50-\mathrm{mL}$ syringe. Finally, the 6-mm probe of a VCS $130 \mathrm{~PB}$ ultrasonic processor (Sonics and Materials Inc, Newtown, CT) was placed at the air-liquid interface, and the solution was sonicated at $130 \mathrm{~W}$ for 5 minutes to form the NBs (Figure 1). Another sample of MBs was prepared as a control using the same formula, but without DPPA, Pluronic F-68, or glycerol.

The diameters of the bubbles varied widely (from $100 \mathrm{~nm}$ to $3000 \mathrm{~nm}$ ); thus, a purification of NBs between $200 \mathrm{~nm}$ and $700 \mathrm{~nm}$ in diameter was necessary. A low-speed centrifugation $(50 \times g, 5$ minutes $)$ separated large bubbles from the suspension as a thin layer, which was discarded. Centrifugation at a higher speed $(805 \times g, 30$ minutes $)$ was performed after the suspension was transferred to a $15-\mathrm{mL}$ centrifuge tube. Small NBs were collected after removing the lower liquid layer, which contained phospholipid fragments and liposomes. Finally, the NBs were resuspended in $4 \mathrm{~mL}$ phosphate-buffered saline (PBS) and stored at $4{ }^{\circ} \mathrm{C}$.

The NB and MB bubble concentrations were determined using a hemacytometer. A drop of DiI-labeled sample was transferred to a hemacytometer and observed using a Carl Zeiss Aviox-1 inverted fluorescence microscope (Carl Zeiss, Oberkirchen, Germany). A fluorescent compound, Dil, was used to detect bubbles that were not detectable at visible wavelengths. Three pairs of images (one fluorescent and one bright field image of each field) at different fields were 


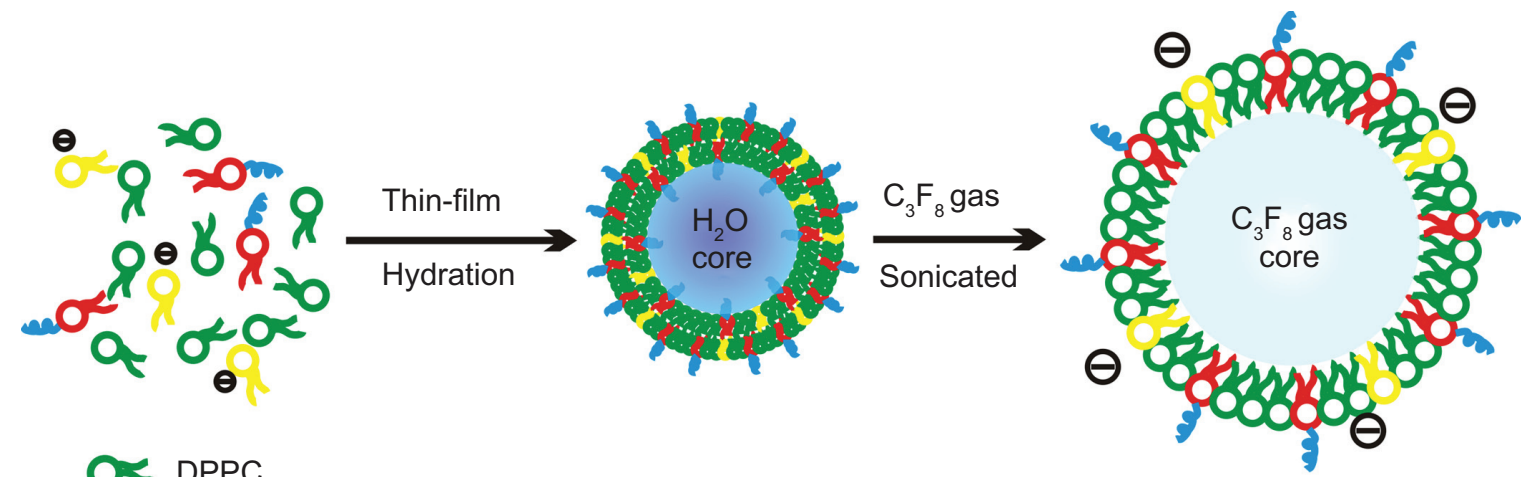

Liposome

Nanobubble

Figure I Formation and structural transitions of nanobubbles for ultrasonic imaging and tumor targeting.

Abbreviations: DPPA, I,2-dipalmitoyl-sn-glycero-3-phosphate; DPPC, I,2-dipalmitoyl-sn-glycero-3-phosphocholine; PEG-DSPE, I,2-distearoyl-sn-glycero-3-phosphoethanolamine$\mathrm{N}$-[biotinyl(polyethylene glycol)2000].

acquired (400×). The boundary of each bubble-counting region was confirmed in the bright field image using the hemacytometer. The number of bubbles in each region was then counted using WCIF ImageJ software (v1.37; National Institutes of Health, Bethesda, MA). Finally, the concentration of the sample (bubbles $/ \mathrm{mL}$ ) was calculated using the same cell-counting method. All measurements were carried out in triplicate.

\section{Particle-sizing and zeta potential measurements}

The particle sizes were measured using dynamic light scattering (DLS) with a laser wavelength of $660 \mathrm{~nm}$ at an angle of $90^{\circ}$ using the 90Plus Multi-angle Particle Sizing Option (Brookhaven Instruments Corporation, Holtsville, NY). Ten microliters of sample and $90 \mu \mathrm{L}$ of PBS were mixed in sample wells before measuring the particle sizes at $25^{\circ} \mathrm{C}$. The zeta potential of each sample was measured using a Zeta Plus Analyzer (Brookhaven Instruments Corporation) to determine the electrophoretic light scattering at $25^{\circ} \mathrm{C}$. All samples used for zeta potential measurements were prepared at the same concentration as those used for particle sizing. The particle size and zeta potential of each sample were measured five times.

\section{Scanning electron microscopy}

To visualize the structure of the NBs, scanning electron microscopy (SEM) images were recorded. A drop of sample on a dust-free foil was placed in a desiccator. After the solvent evaporated, each sample was gold sputter-coated for 5 minutes. A field emission scanning electron microscope (JSM-6330F; JEOL, Tokyo, Japan) was used with a gun acceleration voltage of $3.0 \mathrm{kV}$ and a working distance of $9.7 \mathrm{~mm}$.

\section{Biocompatibility tests Cytotoxicity assay}

To confirm good biocompatibility in vitro, we selected the mouse prostatic cancer cell line RM-1 to evaluate the cytotoxicity of the NBs using the MTT assay. All experiments were conducted in triplicate. The cells were seeded in 96-well plates at a density of 5000 cells/well and then cultured for 24 hours in $100 \mu \mathrm{L}$ of RPMI-1640 medium containing $10 \%$ fetal bovine serum (FBS) in a humidified atmosphere with $5 \% \mathrm{CO}_{2}$. The cells were then incubated for 24 hours in the same volume of fresh medium with various phospholipid concentrations $(0.5-2500 \mu \mathrm{g} / \mathrm{mL})$; the medium was then replaced with $100 \mu \mathrm{L}$ of fresh medium containing $10 \mu \mathrm{L}$ of MTT solution $(5 \mathrm{mg} / \mathrm{mL})$, and the cells were subsequently incubated for 4 hours. Dimethyl sulfoxide $(100 \mu \mathrm{L})$ was added to dissolve the substrate after the MTT-containing supernatant was discarded. After gentle agitation for 5 minutes, the absorbance of each well at $494 \mathrm{~nm}$ was recorded using an Infinite F200 multimode plate reader (Tecan, Männedorf, Switzerland).

\section{Hemolysis test}

Blood was obtained from the main abdominal artery of Sprague-Dawley rats and was collected in a tube that contained heparin sodium $(15 \mathrm{UI} / \mathrm{mL})$. Each $4 \mathrm{~mL}$ of rat blood was mixed with $5 \mathrm{~mL}$ of normal saline. Then, $0.2 \mathrm{~mL}$ of blood diluent was added to $10 \mathrm{~mL}$ of normal saline as a negative control, and $0.2 \mathrm{~mL}$ of blood diluent was added to $10 \mathrm{~mL}$ of double-distilled water as a positive control. The same erythrocyte concentration was used for all phospholipid concentrations $(0.5-2500 \mu \mathrm{g} / \mathrm{mL})$. The solutions were incubated in a $37^{\circ} \mathrm{C}$ water bath for 1 hour. The degree of hemolysis 
was determined spectrophotometrically at $540 \mathrm{~nm}$ using an Infinite F200 multimode plate reader (Tecan).

\section{In vitro ultrasound imaging}

To compare the ultrasonic imaging ability of the NBs with MBs, in vitro ultrasound imaging experiments were carried out; $1 \mathrm{~mL}$ of $\mathrm{NB}$ and $\mathrm{MB}$ suspension at various bubble concentrations (from $1.0 \times 10^{5}$ to $6.4 \times 10^{6}$ bubbles $/ \mathrm{mL}$ ) was added to the sample wells of a custom-made $2 \%$ (w/v) agarose mold (Figure 2). Another sample of NB at the concentration of $8.0 \times 10^{5}$ bubbles $/ \mathrm{mL}$ was exposed to high-power ultrasound $\left(1 \mathrm{MHz}, 0.78 \mathrm{~W} / \mathrm{cm}^{2}\right)$ for 30 seconds by using a self-made low-frequency therapeutic ultrasound system (Chongqing, China) for NB destruction experiment. A clinical ultrasound scanner (Acuson Sequoia 512; Siemens, Malvern, PA) system with a 15 L8-S high-frequency linear transducer was used. The transmitted power was $-18 \mathrm{db}$, which corresponded to an MI of 0.10 . The contrast pulse sequencing (CPS) gain was $0 \mathrm{db}$, and the focal zone was placed at a depth of $1.5 \mathrm{~cm}$, which was at the center of the sample well. Three images were taken for each sample.

Image analysis was performed using ImageJ software to analyze the gray-scale values of the samples. Circular regions of interest (ROIs) were outlined in each sample well. The quantitative grey-scale ultrasonic intensity of the samples was normalized to that of gas-free water. The intensity value was defined as the gray-scale value ratio of contrast agent to gas-free water.

\section{In vivo contrast-enhanced ultrasound imaging}

The in vivo imaging capability of the NB contrast agents was evaluated using Sprague-Dawley rats. Each rat was

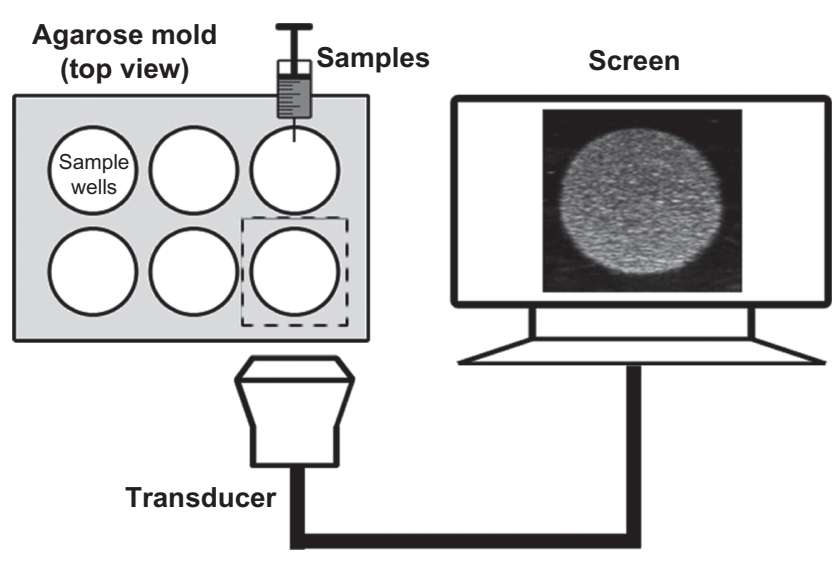

Figure 2 Custom-made $2 \%(w / v)$ agarose mold for use with in vitro ultrasound imaging and the in vitro experimental setup. anesthetized with $300 \mathrm{mg} / \mathrm{kg}$ of $10 \%$ chloral hydrate by intraperitoneal injection. The animals were placed on a warm blanket to maintain their body temperature within normal range. The NB samples $\left(10^{9}\right.$ bubbles $\left./ \mathrm{mL}\right)$ were intravenously injected at a dosage of $0.1 \mathrm{~mL} / \mathrm{kg}$. Various organs, such as the heart, liver and kidneys, were imaged transabdominally using a broadband 15L8-S high-frequency linear transducer in CPS mode with an MI of 0.19. All animal experiments were carried out in accordance with the procedures and guidelines of the Institutional Animal Care and Use Committee and were approved by the Animal Experiment Committee and Biosafety Committee at Sun Yat-Sen University of Medical Science.

\section{In vivo passive tumor-targeting ability}

RM-1 cells were transplanted into BALB/c nude mice for passive tumor-targeting imaging experiments. The cells were cultured in RPMI-1640 medium supplemented with $10 \%$ FBS (GIBCO, Carlsbad, CA) and incubated at $37^{\circ} \mathrm{C}$ in a humidified atmosphere of $5 \% \mathrm{CO}_{2}$ in air. A total of six $\mathrm{BALB} / \mathrm{c}$ nude mice (5 weeks, $18-23 \mathrm{~g}$ ) were examined. The cells $\left(10^{6}\right)$ were resuspended in $0.1 \mathrm{~mL}$ of PBS and subcutaneously injected into the dorsal scapular area on the right side for tumor xenografts. All in vivo experiments began when the tumors reached a diameter of $0.8-1.2 \mathrm{~cm}$.

Mice were anesthetized with $10 \%$ chloral hydrate and fixed on a plate before ultrasonic imaging. The ultrasonic transducer was fixed on an iron support stand. The distance between the transducer and the tumor was approximately $2 \mathrm{~cm}$, and the space between them was filled with an adequate quantity of ultrasonic transmission gel. MBs and NBs were used in the same mice to compare the performance of NBs to that of MBs. MBs $\left(6.0 \times 10^{6}\right)$ were suspended in $50 \mu \mathrm{L}$ of PBS and injected into the tail vein. Ultrasonic images were acquired using a 7-MHz transducer in CPS mode, and the acoustic focal zone was placed at the center of the tumor at the largest transverse cross-section. A 10-minute CPS imaging sequence was acquired, and further images were recorded every 30 seconds for 1.5 hours. After the MB experiments, the bubbles were allowed to clear from the circulation of the mouse for 2 hours. Finally, the NBs were studied using the same bubble concentration and imaging protocol.

All digital clips and images were stored for offline examination. Gray-scale images were analyzed using ImageJ. The quantitative gray-scale ultrasonic intensity of each image was defined as the ratio of post-contrast agent injection to pre-contrast agent injection. A time-intensity curve for each mouse was created, and three important parameters were 
analyzed statistically: time to peak (TTP), peak intensity (PI), and half-time of washout (HT).

\section{Confocal laser scanning microscopy examination}

To confirm that the NBs were small enough to pass through the endothelial gaps of tumors, we used confocal laser scanning microscopy (CLSM) to determine the location of red fluorescently dyed NBs in vivo; this technique is regarded as the gold standard. Tumor-carrying mice were randomly separated into two groups: NB and MB injection. A total of $250 \mu \mathrm{L}\left(2.5 \times 10^{7}\right.$ bubbles $\left./ \mathrm{mL}\right)$ of DiI-labeled NBs or MBs were injected into the tail vein of each mouse. To clear the labeled bubbles from circulation, the heart was perfused with $0.9 \%$ normal saline 3 hours after bubble injection. The tumors and muscles of the right thigh (used as negative controls because the capillaries of skeletal muscle are continuous) were immediately extracted for sectioning into $5-\mu \mathrm{m}$ slices. Frozen sections were stained in a solution of $2 \mu \mathrm{g} / \mathrm{mL}$ Hoechst 33342 for 30 minutes to mark the nucleus. Images were recorded using an Olympus PV1000-IX81 Confocal Microscope (Olympus, Tokyo, Japan). DiI and Hoechst 33342 were excited at 550 and $352 \mathrm{~nm}$, respectively, and the emissions were recorded at 565 and $455 \mathrm{~nm}$, respectively.

\section{Statistical analysis}

The unpaired Student's $t$-test was performed for all comparisons. All data are expressed as the mean \pm the standard errors of the mean (SEM). The data were statistically analyzed with SPSS software (version 13.0; SPSS Inc, Chicago, IL) using one-factor analysis of variance. A $P$ value $<0.05$ was considered statistically significant. All statistical tests were two-tailed.

\section{Results}

\section{Characterization of the bubbles}

The average diameter of the nanoscale ultrasound contrast agent (NBs) was $436.8 \pm 5.7 \mathrm{~nm}(\mathrm{n}=5)$ (Figure $3 \mathrm{~A})$, and that of the control MBs was $1220 \pm 65 \mathrm{~nm}(\mathrm{n}=5)$ (Figure 3B). Zeta potential measurements showed that the NBs had a net negative charge of $-18.36 \pm 0.81 \mathrm{mV}(\mathrm{n}=5)$, while that of the MBs was $-2.93 \pm 0.42 \mathrm{mV}(\mathrm{n}=5)$. The bubble concentrations of the NBs and MBs were $(1.22 \pm 0.16) \times 10^{9}$ bubbles $/ \mathrm{mL}$ $(\mathrm{n}=3)$ and $(5.64 \pm 0.19) \times 10^{8}$ bubbles $/ \mathrm{mL}(\mathrm{n}=3)$, respectively. The negative charge was due to the presence of the anionic phospholipid DPPA, which helped to avoid physical aggregation of the bubbles and restricted the size of the NBs. Surface morphology and size distribution were viewed using SEM (Figure 3C), and the results showed that the NBs were small, spherical, and nonaggregating. The size distribution of the NBs was between $250-500 \mathrm{~nm}$, which was similar to that measured using DLS.

\section{Biocompatibility tests}

The cytotoxicity of the NBs was evaluated using the MTT assay and the mouse prostatic cancer cell line RM-1. Figure 4 shows the cytotoxicity of the NBs after incubation for 24 hours. The MTT results indicate that the NBs had no obvious cytotoxicity toward this cell line within the concentrations used for in vivo ultrasound imaging with phospholipids $(0.5-5 \mu \mathrm{g} / \mathrm{mL})$. At higher phospholipid concentrations (higher than $10 \mu \mathrm{g} / \mathrm{mL}$ ), their cytotoxicity increased rapidly. In vitro hemolysis tests were carried out spectrophotometrically. As shown in Figure 4, the presence of lower concentrations of NBs had no effect on the rate of hemolysis. However, at phospholipid concentrations higher than $50 \mu \mathrm{g} / \mathrm{mL}$, hemolysis occurred in a dosedependent manner.

\section{In vitro and in vivo contrast enhancement abilities of NBs}

Ultrasound images were acquired at various bubble concentrations (Figure 5A) using diagnostic high-frequency ultrasound $(7 \mathrm{MHz})$. The results showed that as the bubble concentration increased, the ultrasonic signals of both the NBs and MBs increased. NBs exhibited an
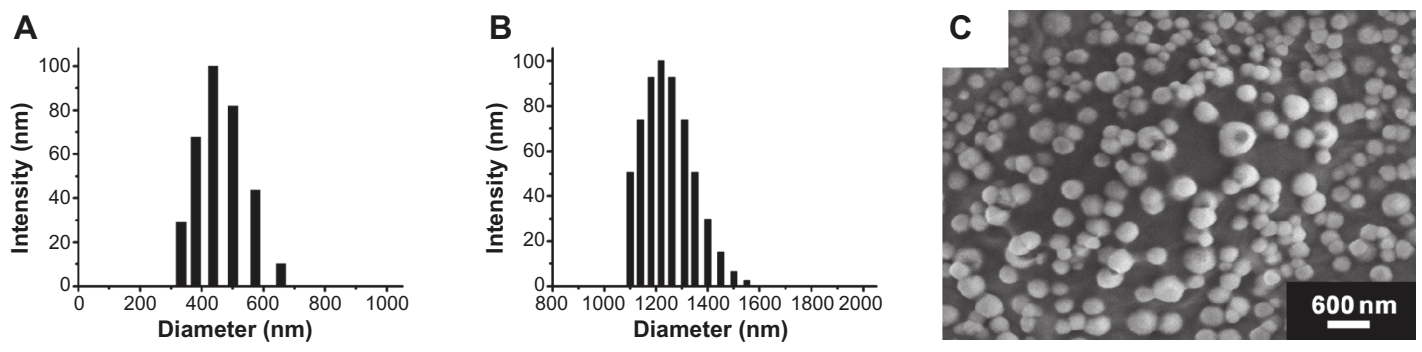

Figure 3 Particle size and morphology of the nanobubbles. The diameter distribution was measured using dynamic light scattering in the nanobubbles (A) and microbubbles (B). The surface morphology of the nanobubbles was visualized using scanning electron microscopy (C). 


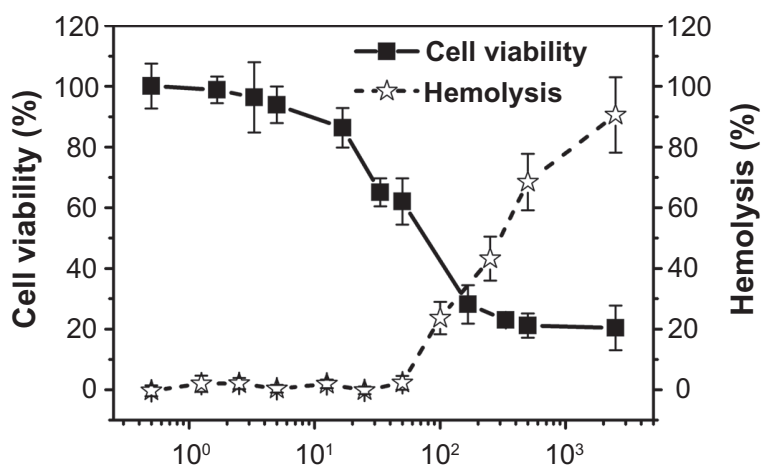

Phospholipid concentration $(\mu \mathrm{g} / \mathrm{mL})$

Figure $\mathbf{4}$ In vitro cytotoxicity of various concentrations of nanobubbles in RM-I cells determined using the MTT assay. The hemolysis rate of erythrocyte suspensions incubated in the presence of various amounts of nanobubbles.

ultrasonic contrast enhancement ability similar to that of MBs (Figure 5B). No statistical differences were observed between the signal enhancement from NBs and that of MBs $(P=0.134)$. After high-power ultrasound exposure, gray-scale intensity decreased as NBs were destroyed (Figure 5C).

In the in vivo studies, CPS-mode gray-scale images were obtained before and after the administration of NB contrast agent to Sprague-Dawley rats (Figure 6). The heart, liver, and kidneys showed excellent enhancement several seconds after intravascular administration of the NBs.

\section{Imaging of tumors based on passive tumor targeting}

CPS-mode imaging was carried out on six tumor-carrying mice. No animals died during the experiment. Figure 7 shows a representative set of images of the contrast enhancement provided by NBs (Figure 7A) and control MBs (Figure 7B) over time $(0,0.5,1,5,10$, and 15 minutes $)$. The tumor imaging results showed that the NBs considerably improved contrast enhancement. The enhancement obtained using NBs lasted for approximately 1 hour, whereas that obtained using the control MBs lasted for only 15 minutes. At 15 minutes after injection, the gray-scale intensity of the NBs was significantly higher than that of the control MBs $(P<0.001)$.

Representative time-intensity curves show the increases in the contrast ratio of the intensity produced by the NBs and control MBs over time (Figure 7C). The slope of the NB line is less steep than that of the control MB line. With the NBs, the TTP was later $(P=0.015)$, the PI was lower $(P=0.027)$, and the HT was longer $(P=0.001)$ than the corresponding values for the control MBs (Table 1).
A

$6.4 \times 10^{6}$ bubbles $/ \mathrm{mL}$

$3.2 \times 10^{6}$ bubbles $/ \mathrm{mL}$

$8.0 \times 10^{5}$ bubbles $/ \mathrm{mL}$

$1.0 \times 10^{5}$ bubbles $/ \mathrm{mL}$
NBs

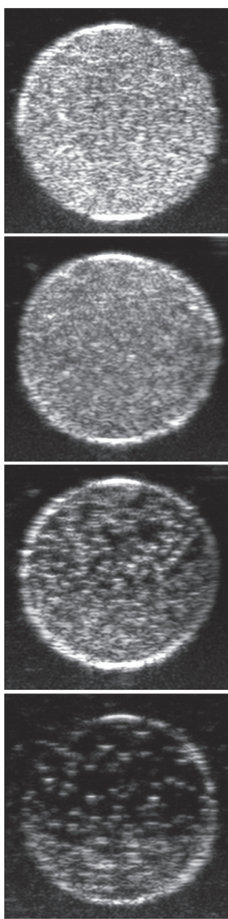

MBs

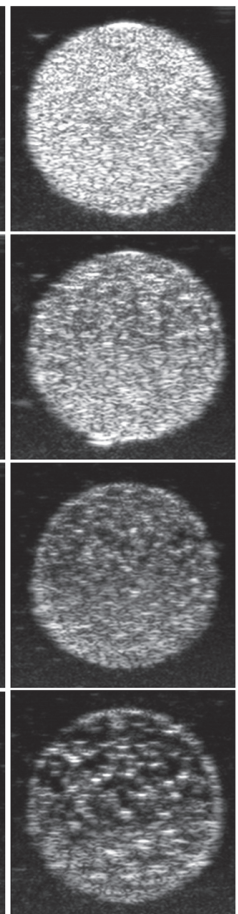

B

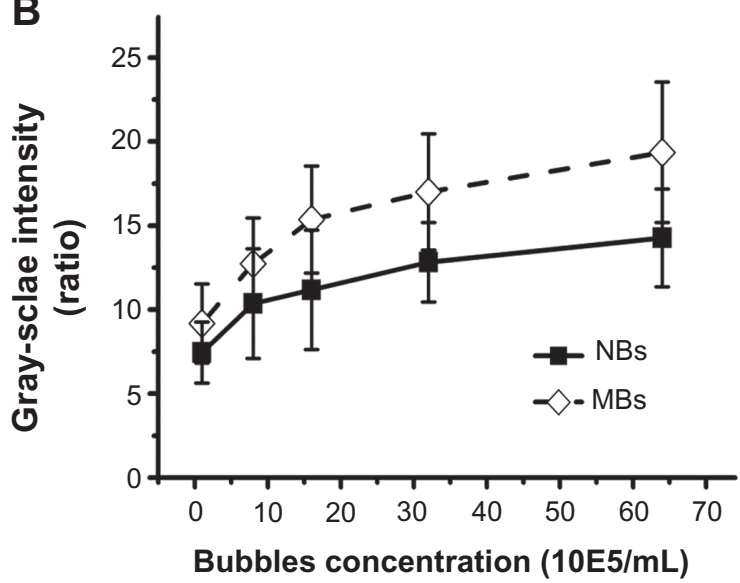

C

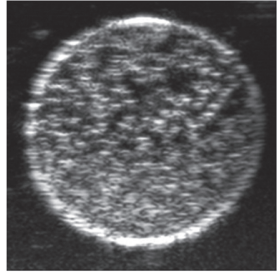

Predestruction

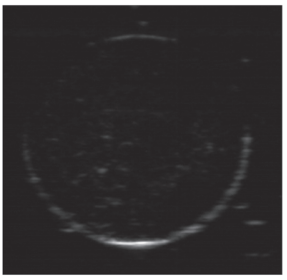

Postdestruction

Figure 5 Representative in vitro ultrasonic images (A) of nanobubbles (NBs) and microbubbles (MBs) at high-frequency diagnostic ultrasound in contrast pulse sequencing mode. (B) Quantitative gray-scale ultrasonic intensity (ratio change over gas-free water). The initial gray-scale value was obtained from gas-free water in the sample wells. NBs presented similar gray-scale intensity to $\mathrm{MBs}$ at $7 \mathrm{MHz}(P=0.134)$. (C) Pre- and postdestruction of NBs low-frequency ultrasound exposure. 


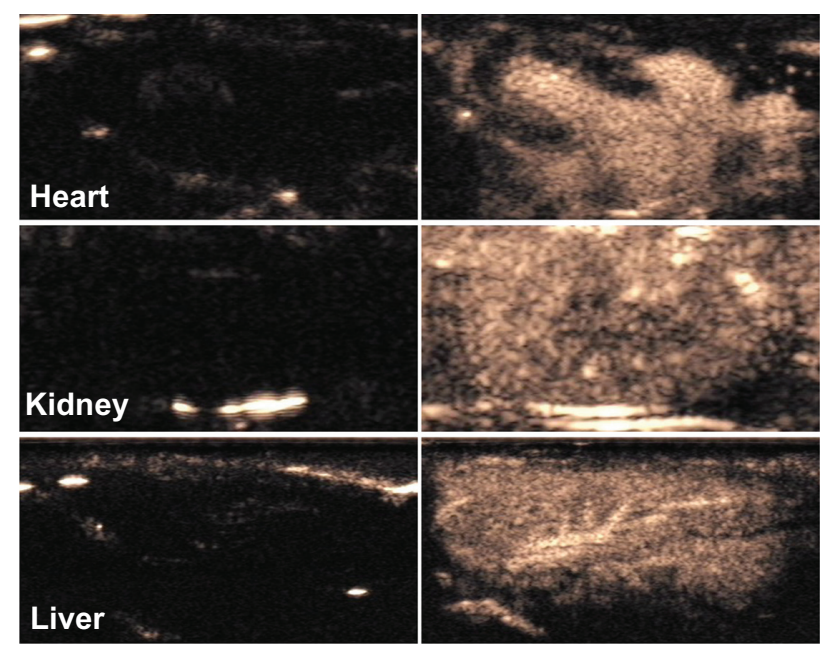

Figure 6 Contrast pulse sequencing-mode images of various organs of normal rats. Images after nanobubble injection (right) showed obvious contrast enhancement in the heart, kidney and liver of Sprague-Dawley rats compared with preinjection images (left).

\section{CLSM experiments}

Under CLSM, the distributions of the DiI-labeled NBs or MBs in the frozen sections of the tumors and skeletal muscle were apparent (Figure 8). In tumors, a considerable number of DiI-labeled NBs were present in the intercellular space (Figure 8A), whereas the NBs could barely be observed in skeletal muscle sections (Figure 8C). In the control, rare DiIlabeled MBs were observed in tumors and skeletal muscle (Figure 8B and D).

\section{Discussion}

Recently, nanoscale ultrasound contrast-enhanced agents with various shells (polymers or phospholipids) and cores (gas, liquid, or solid) have been fabricated. Good contrastenhanced effects have been observed in a number of reported studies, and phospholipid-shell and gas-core NBs have shown optimal contrast-enhancing abilities. ${ }^{16-19}$ However, studies in vivo have focused on the contrast enhancement abilities of these agents in normal organs or tumors, not on the potential of NBs for the passive targeting of tumors. As a result, the purpose of this work was to fabricate nanosized, phospholipidshelled NBs with high ultrasonic-imaging efficiency and of a sufficiently small size to pass through the pores of tumor vasculature and achieve passive tumor targeting. Herein, NBs that were demonstrated to have small diameters using DLS and SEM were evaluated for their ultrasound imaging ability in vitro and their delayed imaging ability in tumor tissues.

A key factor in producing small NBs was the presence of Pluronic F-68, which stabilizes the NBs, controls their size, and interacts with lipid shells to change the lipid fluidity or
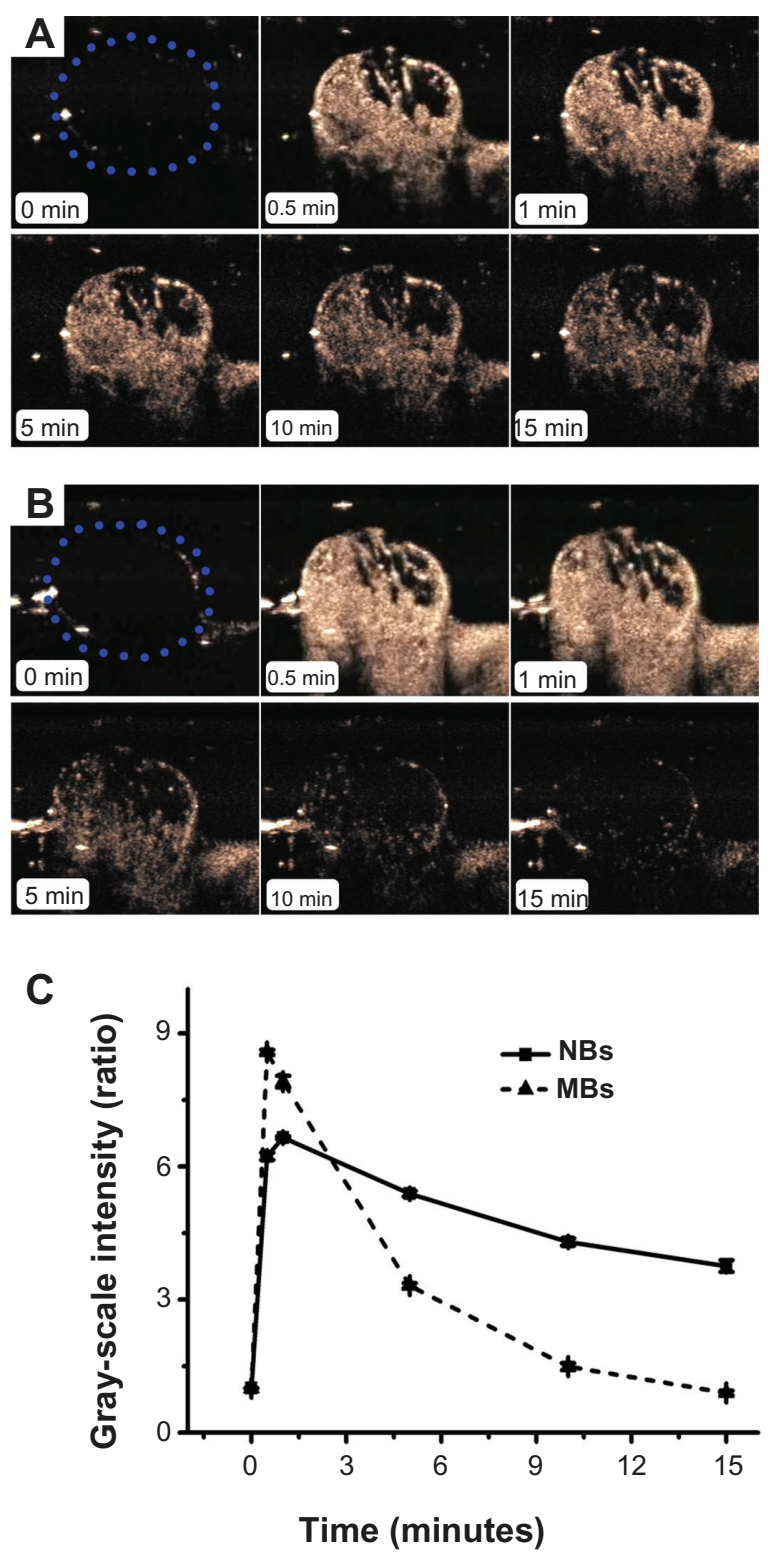

Figure 7 In vivo passive tumor targeting. Representative subcutaneous tumor images before (blue dotted line) and after the injection of nanobubbles (NBs) (A) compared with microbubbles (MBs) (B) at various time points $(0,0.5,1,5,10$, and 15 minutes). The corresponding time-intensity curve of tumor enhancement after injection of the contrast agent $(\mathbf{C})$.

bubble elastic modulus. ${ }^{22}$ One of the components, the anionic phospholipid DPPA, equipped the NBs with negatively charged membranes to avoid aggregation and enhance the stability of the bubbles due to electrostatic repulsive forces. SEM is the gold standard for determining the diameter distribution and morphology of NBs. The results of SEM and DLS showed that the NBs were small $(<500 \mathrm{~nm})$ and spherical, which implies that the small NBs with the large bubbles removed responded well to ultrasound.

The NBs were composed of phospholipids that are used in the cell cytomembrane and are known to be low-toxicity 
Table I Quantitative parameters of $\mathrm{NBs}$ and $\mathrm{MBs}$ in in vivo tumor ultrasound imaging (mean \pm SEM)

\begin{tabular}{llll}
\hline & TTP (seconds) & PI (ratio) & HT (seconds) \\
\hline NBs & $165.0 \pm 15.1$ & $6.9 \pm 0.8$ & $1265.0 \pm 60.3$ \\
MBs & $24.2 \pm 2.5$ & $8.4 \pm 0.7$ & $310.0 \pm 20.7$ \\
$t$ & 3.660 & -3.096 & 7.222 \\
$P$ & 0.015 & 0.027 & 0.001 \\
\hline
\end{tabular}

Abbreviations: $\mathrm{HT}$, half-time to washout; MBs, microbubbles; NBs, nanobubbles; $\mathrm{PI}$, peak intensity; SEM, scanning electron microscopy; TTP, time to peak.

materials. The results of the MTT assay and the hemolysis test, which were used to determine the cytotoxicity of the NBs, confirmed their biological safety. At commonly used phospholipid concentrations, the NBs were safe for use in cell studies and in vivo ultrasound imaging.

The NBs exhibited similar echogenic ability to the control MBs in vitro using high-frequency diagnostic ultrasound. This similarity likely resulted from two factors. First, lipid shells are easier to form and yield a very echogenic and elastic bubble. ${ }^{23}$ Highly compressible shells play an important role in the echogenicity of NBs by allowing high scattering. ${ }^{24}$ Second, the CPS mode of the Sequoia 512 scanning instrument is not only a harmonic imaging mode, but also a summation of corresponding echoes from three pulses with different amplitudes and phases. ${ }^{25}$ Although the resonant frequency of the NBs was much higher than that of the clinical diagnostic ultrasound, the high scattering may enhance the acoustic imaging impact of these NBs.

The results of in vivo ultrasonic experiments used to examine the passive targeting imaging of tumors suggested that NBs are retained in the tumor tissue for longer periods than MBs. All parameters of the time-intensity curve supported the imaging results. The TTP was later $(P=0.015)$, the PI was lower $(P=0.027)$, and the HT was longer $(P=0.001)$ in the NB group than the corresponding values for the control MB group in tumor areas; all of these results stemmed from the small size of the NBs. First, the small diameter helped the bubbles to permeate the tumor vasculature and remain in the tumor tissue. ${ }^{1}$ As time passed, greater numbers of NBs passed through the endothelial gaps and were retained. This allowed the echogenicity to remain strong for much longer periods than the MBs, which were almost cleared after 10 minutes. Second, some time is necessary for the permeation and aggregation of the NBs; this requirement explains why the TTP of the NBs was later than that of the control MBs, which were too large to fit through the endothelial gaps. Third, because of their small size, the NBs were expected to absorb high-frequency ultrasound to a greater degree than their scattering at the fundamental frequency; ${ }^{26}$ thus, the NBs exhibited a lower peak intensity in this study. Finally, their small size and PEGylated lipid shells helped the NBs escape from the mononuclear phagocyte system. ${ }^{27,28}$

CLSM imaging revealed the location of the NBs after intravenous injection. The majority of tumors have a pore cutoff size between 380 and $780 \mathrm{~nm},{ }^{16,29}$ which is the foundation of the molecular imaging of tumors using passive tumor targeting. ${ }^{30}$ In the present study, DiI-labeled NBs penetrated through interendothelial gaps and accumulated in the tumor, and red fluorescence was observed in the images. However,

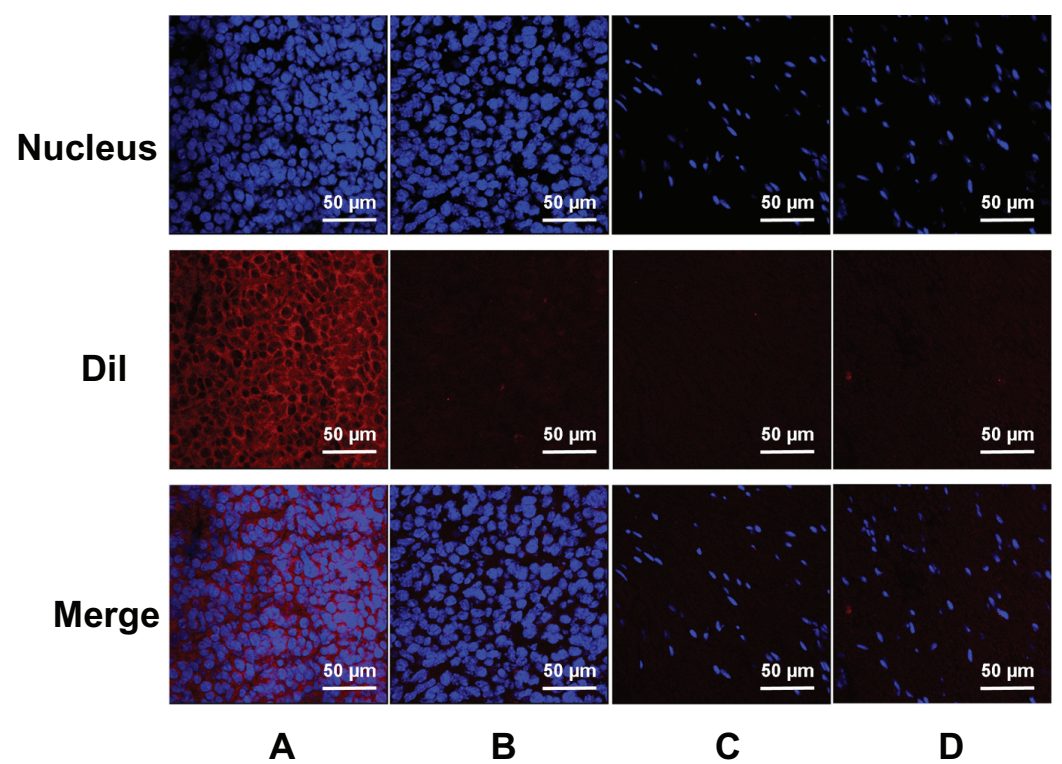

Figure 8 Confocal laser-scanning microscopy images of frozen sections after nuclear labeling. A considerable number of Dil-labeled nanobubbles are observed in the intercellular space (A), whereas Dil-labeled microbubbles are hardly visible in tumors (B). Both Dil-labeled nanobubbles and microbubbles were difficult to detect in skeletal muscle (C and $\mathbf{D})$. 
the MBs were absent from the tumor tissue as demonstrated by the almost complete absence of DiI red fluorescence. These phenomena could explain the ultrasonic imaging performance. At the later stage of ultrasound contrast-enhanced imaging, NBs passed through the pores in tumor vessels and accumulated in the tumor; therefore, the contrast enhancement remained for a much longer period than when using the MBs, which do not pass through. The inter-endothelial gaps of normal tissue are less than $7 \mathrm{~nm},{ }^{31}$ and neither NBs nor MBs can pass through gaps of this size. As a result, there was passive targeting of the NBs to tumors.

\section{Conclusion}

The basic requirement of tumor imaging and targeting by ultrasound is that the particle size of the ultrasound contrastenhanced agents be small. However, a nanoscale particle size reduces the ultrasonic imaging ability in clinical diagnostic ultrasound applications. How this contradiction is solved to realize tumor targeting is an important topic in the use of ultrasound in molecular imaging. In this work, we fabricated a nano-sized lipid NB contrast-enhanced ultrasound agent and evaluated its echogenic ability in vitro and in vivo. Its ability to passively target tumors was confirmed by in vivo ultrasonic imaging and CLSM. Their characteristics suggest that NBs may be applicable to ultrasonic molecular imaging and tumor-targeting therapy. Potential applications for NBs include contrast-enhanced imaging and drug/gene delivery to tumors.

\section{Acknowledgments}

This work was financially supported by the National Natural Science Foundation of China (30870717, 81071167, 81000191, and 50830107).

\section{Disclosure}

The authors report no conflicts of interest in this work.

\section{References}

1. Iyer AK, Khaled G, Fang J, Maeda H. Exploiting the enhanced permeability and retention effect for tumor targeting. Drug Discov Today. 2006;11(17-18):812-818.

2. Skinner SA, Tutton PJ, O'Brien PE. Microvascular architecture of experimental colon tumors in the rat. Cancer Res. 1990;50(8): 2411-2417.

3. Matsumura Y, Maeda H. A new concept for macromolecular therapeutics in cancer chemotherapy: mechanism of tumoritropic accumulation of proteins and the antitumor agent smancs. Cancer Res. 1986;46(12 Pt 1): 6387-6392.

4. Dai J, Zou S, Pei Y, Cheng D, Ai H, Shuai X. Polyethylenimine-grafted copolymer of poly(L-lysine) and poly(ethylene glycol) for gene delivery. Biomaterials. 2011;32(6):1694-1705.
5. Yang X, Zhu B, Dong T, Pan P, Shuai X, Inoue Y. Interactions between an anticancer drug and polymeric micelles based on biodegradable polyesters. Macromol Biosci. 2008;8(12):1116-1125.

6. Cao N, Cheng D, Zou S, Ai H, Gao J, Shuai X. The synergistic effect of hierarchical assemblies of siRNA and chemotherapeutic drugs co-delivered into hepatic cancer cells. Biomaterials. 2011;32(8):2222-2232.

7. Wilson SR, Burns PN. Microbubble-enhanced US in body imaging: what role? Radiology. 2010;257(1):24-39.

8. Deshpande N, Needles A, Willmann JK. Molecular ultrasound imaging: current status and future directions. Clin Radiol. 2010;65(7): $567-581$.

9. Danila M, Popescu A, Sirli R, Sporea I, Martie A, Sendroiu M. Contrast enhanced ultrasound (CEUS) in the evaluation of liver metastases. Med Ultrason. 2010;12(3):233-237.

10. Cavalieri F, Zhou M, Ashokkumar M. The design of multifunctional microbubbles for ultrasound image-guided cancer therapy. Curr Top Med Chem. 2010;10(12):1198-1210.

11. Gessner R, Dayton PA. Advances in molecular imaging with ultrasound. Mol Imaging. 2010;9(3):117-127.

12. Sanna V, Pintus G, Bandiera P, et al. Development of polymeric microbubbles targeted to prostate-specific membrane antigen as prototype of novel ultrasound contrast agents. Mol Pharm. 2011;8(3): $748-757$.

13. Wu J, Leong-Poi H, Bin J, et al. Efficacy of contrast-enhanced US and magnetic microbubbles targeted to vascular cell adhesion molecule-1 for molecular imaging of atherosclerosis. Radiology. 2011;260(2): 463-471.

14. Messina SA, Aras O, Dilsizian V. Delayed recovery of fatty acid metabolism after transient myocardial ischemia: a potential imaging target for "ischemic memory". Curr Cardiol Rep. 2007;9(2):159-165.

15. Weller GE, Lu E, Csikari MM, et al. Ultrasound imaging of acute cardiac transplant rejection with microbubbles targeted to intercellular adhesion molecule-1. Circulation. 2003;108(2):218-224.

16. Oeffinger BE, Wheatley MA. Development and characterization of a nano-scale contrast agent. Ultrasonics. 2004;42(1-9):343-347.

17. Kim C, Qin R, Xu JS, Wang LV, Xu R. Multifunctional microbubbles and nanobubbles for photoacoustic and ultrasound imaging. J Biomed Opt. 2010;15(1):10510.

18. Rapoport N, Gao Z, Kennedy A. Multifunctional nanoparticles for combining ultrasonic tumor imaging and targeted chemotherapy. J Natl Cancer Inst. 2007;99(14):1095-1106.

19. Marxer EE, Brussler J, Becker A, et al. Development and characterization of new nanoscaled ultrasound active lipid dispersions as contrast agents. Eur J Pharm Biopharm. 2011;77(3):430-437.

20. Xing Z, Wang J, Ke H, et al. The fabrication of novel nanobubble ultrasound contrast agent for potential tumor imaging. Nanotechnology. 2010;21(14):145607.

21. Watanabe $\mathrm{Y}$, Horie S, Funaki Y, et al. Delivery of Na/I symporter gene into skeletal muscle using nanobubbles and ultrasound: visualization of gene expression by PET. J Nucl Med. 2010;51(6):951-958.

22. Krupka TM, Solorio L, Wilson RE, Wu H, Azar N, Exner AA. Formulation and characterization of echogenic lipid-Pluronic nanobubbles. Mol Pharm. 2010;7(1):49-59.

23. Ferrara K, Pollard R, Borden M. Ultrasound microbubble contrast agents: fundamentals and application to gene and drug delivery. Annu Rev Biomed Eng. 2007;9:415-447.

24. Flegg MB, Poole CM, Whittaker AK, Keen I, Langton CM. Rayleigh theory of ultrasound scattering applied to liquid-filled contrast nanoparticles. Phys Med Biol. 2010;55(11):3061-3076.

25. Anderson $\mathrm{CR}, \mathrm{Hu} \mathrm{X}$, Zhang $\mathrm{H}$, et al. Ultrasound molecular imaging of tumor angiogenesis with an integrin targeted microbubble contrast agent. Invest Radiol. 2011;46(4):215-224.

26. Sirsi S, Feshitan J, Kwan J, Homma S, Borden M. Effect of microbubble size on fundamental mode high frequency ultrasound imaging in mice. Ultrasound Med Biol. 2010;36(6):935-948.

27. Brigger I, Dubernet C, Couvreur P. Nanoparticles in cancer therapy and diagnosis. Adv Drug Deliv Rev. 2002;54(5):631-651. 
28. Diaz-Lopez R, Tsapis N, Santin M, et al. The performance of PEGylated nanocapsules of perfluorooctyl bromide as an ultrasound contrast agent. Biomaterials. 2010;31(7):1723-1731.

29. Hobbs SK, Monsky WL, Yuan F, et al. Regulation of transport pathways in tumor vessels: role of tumor type and microenvironment. Proc Natl Acad Sci U S A. 1998;95(8):4607-4612.
30. Weissleder R. Molecular imaging: exploring the next frontier. Radiology. 1999;212(3):609-614.

31. Rapoport NY, Nam KH, Gao Z, Kennedy A. Application of ultrasound for targeted nanotherapy of malignant tumors. Acoust Phys. 2009;55(4-5):594-601.

\section{Publish your work in this journal}

The International Journal of Nanomedicine is an international, peerreviewed journal focusing on the application of nanotechnology in diagnostics, therapeutics, and drug delivery systems throughout the biomedical field. This journal is indexed on PubMed Central, MedLine, CAS, SciSearch $\AA$, Current Contents ${ }^{\circledR} /$ Clinical Medicine,
Journal Citation Reports/Science Edition, EMBase, Scopus and the Elsevier Bibliographic databases. The manuscript management system is completely online and includes a very quick and fair peer-review system, which is all easy to use. Visit http://www.dovepress.com/ testimonials.php to read real quotes from published authors.

Submit your manuscript here: http://www.dovepress.com/international-journal-of-nanomedicine-journal 